\title{
Recovery of Plasma from Blood Products
}

National Cancer Institute

\section{Source}

National Cancer Institute. Recovery of Plasma from Blood Products. NCI Thesaurus.

Code C112948.

A process that derives plasma from single units of whole blood or as a by-product from the preparation of blood components from whole blood. 